
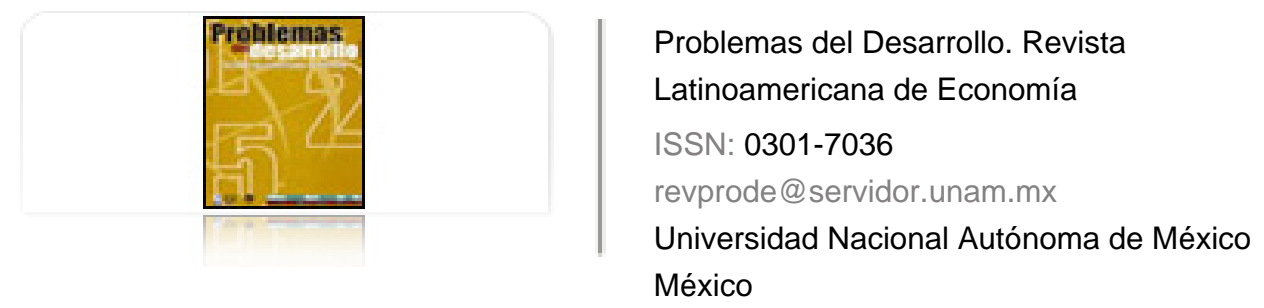

Franco Martínez, Juan Agustín

Economía y agroerosión en el sur de España

Problemas del Desarrollo. Revista Latinoamericana de Economía, vol. 39, núm. 154, julio-septiembre, 2008, pp. 135-156

Universidad Nacional Autónoma de México

Distrito Federal, México

Disponible en: http://www.redalyc.org/articulo.oa?id=11820139007

Cómo citar el artículo

- Número completo

- Más información del artículo

Página de la revista en redalyc.org

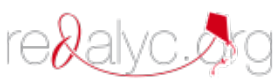

Sistema de Información Científica

Red de Revistas Científicas de América Latina, el Caribe, España y Portugal Proyecto académico sin fines de lucro, desarrollado bajo la iniciativa de acceso abierto 


\title{
ECONOMÍA Y AGROEROSIÓN EN EL SUR DE ESPAÑA
}

\author{
Juan Agustín Franco Martínez*
}

Fecha de recepción: 25 de enero de 2008. Fecha de aceptación: 7 de abril de 2008.

\section{Resumen}

Este artículo estudia el problema agroambiental por la erosión del suelo en el olivar del sur de España, visto como un fenómeno derivado del desarrollo económico. Para ello se revisa la normativa europea sobre el impacto ambiental en el sector agrario como consecuencia de unas políticas macroeconómicas de producción intensiva. Además, se revisa la literatura científica sobre la dimensión económica de los procesos de innovaciones agrarias relativos a la adopción de prácticas de conservación del suelo. Después se realiza un estudio empírico de la productividad económica de una muestra de 582 parcelas de olivar de las provincias andaluzas de Granada y Jaén en 2004, las cuales fueron seleccionadas por sus niveles medios y altos de erosión del suelo.

Palabras clave: productividad económica, erosión del suelo, legislación agroambiental, cuenca mediterránea, olivar.

* Profesor ayudante de la Facultad de Ciencias Económicas y Empresariales en la Universidad de Zaragoza, España. Correo electrónico: franco@unizar.es. 
Abstract

This paper studies the agro-environmental problem caused by the erosion of the soil in southern Spain's olive groves, seen as a phenomenon deriving from economic development. It therefore reviews the European regulations on the environmental impact in the agrarian sector as a consequence of macroeconomic policies favoring intensive production. It also reviews the scientific literature on the economic dimension of agrarian innovations relating to the adoption of soil conservation practices. An empirical study is made of the economic productivity of a sample of 582 olive grove lots in the Andalusian provinces of Granada and Jaén in 2004, selected for their medium and high levels of soil erosion.

Key words: economic productivity, soil erosion, agro-environmental legislation, Mediterranean basin, olive grove.

\section{Résumé}

Cet article étudie le problème d'agro-environnement dû à l'érosion du sol dans les zones d'oliveraies du sud de l'Espagne pris comme un phénomène produit par le développement économique. La norme européenne d'impact sur l'environnement par le secteur agricole y est vue comme conséquence de politiques macroéconomiques de production intensive. Il est procédé à l'examen de la littérature scientifique sur la dimension économique des processus d'innovation agraire en ce qui concerne l'adoption de pratiques de conservation des sols. Ensuite est realisée une étude empirique de la productivité économique d'un échantillon de 582 parcelles d'oliveraies dans les provinces andalouses de Granada y Jaén en 2004, lesquelles furent sélectionnées pour les niveaux moyens ou élevés d'érosion de leurs sols.

Mots clés: productivité économique, érosion du sol, légisation agro-environnemental, bassin méditerranéen, oliveraie.

\section{Resumo}

Este artigo estuda o problema agro ambiental pela erosão do solo no olivar do sul da Espanha, visto como um fenômeno derivado do desenvolvimento econômico. Para isto se revisa a normativa européia sobre o impacto ambiental no setor agrário como conseqüência de umas políticas macroeconômicas de produção intensiva. Também se revisa a literatura científica sobre a dimensão econômica dos processos de inovações agrárias relativos a adoção de práticas de conservação do solo. Depois se realiza um estudo empírico da produtividade econômica de uma mostra de 582 parcelas de olivar das províncias andaluzas de Granada e Jaén em 2004, as quais foram selecionadas por seus níveis médios e altos de erosão do solo.

Palavras chave: produtividade econômica, erosão do solo, legislação agro ambiental, bacia mediterrânea, olivar. 


\section{Introducción}

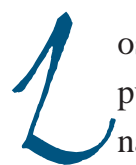

os procesos agrícolas intensivos han aumentado la erosión del suelo hasta el punto de considerarse como una fuente de externalidades negativas que ame-

naza la sostenibilidad agraria, además de reducir el potencial de producción agrícola. Por "externalidad" (también se denomina "efecto externo") se entiende el efecto indirecto de una actividad de producción o de consumo sobre la función de utilidad de un agente económico. Por este motivo se puede afirmar que el mercado no conduce a la eficiencia social si las acciones de los productores o de los consumidores no les afectan sólo a ellos, sino que provocan efectos colaterales (positivos o negativos) sobre otros agentes. Las externalidades surgen en los casos donde no existe un mercado para el intercambio de bienes o servicios, y suelen estar ligadas a temas ambientales (Azqueta, 1994). La erosión de los suelos agrícolas tiene múltiples dimensiones (biológica, física, económica, ecológica, social, etcétera) que deben considerarse juntas, con vistas a una adecuada toma de decisiones. No obstante, el aspecto socioeconómico del problema ha sido ignorado en la mayoría de los estudios técnicos sobre el fenómeno erosivo en la agricultura, predominando las investigaciones de carácter geológico y cartográfico.

La erosión del suelo no sólo se debe a factores naturales, también se debe a la acción del ser humano (cuadro 1). La "erosión natural" constituye un aspecto de la dinámica ecológica, debido a la acción de agentes y procesos naturales que han actuado a lo largo de millones de años. Entre los factores naturales de la erosión del suelo destacan los factores geológicos, geográficos, edáficos, climáticos y biológicos. Por su parte, la "erosión acelerada o artificial" es el resultado de la acción humana, y sus efectos son perceptibles en un periodo mucho menor. El efecto combinado de la erosión natural y humana intensifica esta problemática ambiental. Por ejemplo, el riesgo de erosión por acción del agua es máximo en periodos de lluvias intensas, pues el suelo se encuentra saturado y con escasa cubierta vegetal, por lo que aumenta el movimiento del agua por la superficie terrestre. Además, la incidencia de la erosión por el viento, propia de climas áridos y semiáridos, casi siempre se debe a la disminución de la cubierta vegetal del suelo, bien por sobrepastoreo o a causa de la eliminación de la vegetación para usos domésticos o agrícolas. En el caso de la cuenca mediterránea peninsular existe evidencia geoarqueológica del origen prehistórico de los procesos erosivos derivados de la acción humana como consecuencia de la deforestación por el desarrollo agrícola y ganadero de la zona entre el III y el II milenios A.C. (Gilman y Thornes, 1985; Arteaga et al., 1987). Los procesos erosivos se han intensificado por la acción humana especialmente a partir del siglo XX, debido a la explotación inten- 
siva del suelo mediante nueva maquinaria agrícola, compuestos químicos como herbicidas, fertilizantes, nutrientes, que han permitido aumentar la producción agrícola. Por tanto, la erosión por acción humana es un problema económico y ambiental global cuyo origen es principalmente agrario (Pimentel et al., 1995). Para subsanar esta contradicción entre la conservación de la naturaleza y la explotación de la misma, a fin de satisfacer las necesidades humanas (en apariencia opuestas), se han articulado políticas institucionales que intenten conciliar ambos objetivos en una dinámica de equilibrio sostenible.

Cuadro 1

Principales causas y efectos del problema de la erosión

\begin{tabular}{lll}
\hline & De origen natural & De origen humano \\
\hline Causas & Clima árido & Mal uso de los recursos forestales \\
& Sequías recurrentes & Malas prácticas agrícolas \\
& Incendios intensos y frecuentes & Abandono de cultivos \\
& Otras condiciones climáticas & Exceso de pastoreo \\
& Factores geológicos & Agricultura intensiva \\
& Factores demográficos & Salinización del suelo \\
& Factores edafológicos & Cultivos en pendientes pronunciadas \\
\hline Efectos externos o sociales & Colmatación de embalses & Modificación reservas de recursos \\
& Aparición de surcos, cárcavas... & Grandes inversiones de recuperación \\
& Perturbación ciclo hidrológico & Ruptura equilibrio agro-ganadero \\
& Contribución cambio climático & \\
\hline Efectos endógenos o agrarios & Incremento de la erosión & Reducción superficie y fertilidad \\
& Salinización del suelo & Pérdida de producción \\
& Compactación del suelo & Reducción del agua disponible \\
& Disminución infiltración & Desequilibrios en rendimientos \\
& Incremento escorrentías & Pérdida de ingresos económicos \\
& Reducción biomasa & Aumento de precio de productos \\
Aumento pedregosidad del suelo & Incremento costos de corrección \\
& Degradación biológica del suelo & Abandono tierras de cultivo \\
& Adopción de PCS \\
\hline
\end{tabular}

Fuente: Elaboración propia.

\section{La legislación agroambiental de la UE}

La política agraria común (PAC) se creó en los años sesenta del siglo pasado, en un momento en que Europa era deficitaria en la mayoría de los productos alimenticios. Sus mecanismos se configuraron para resolver esa situación, y su función 
principal era la de apoyar los precios y las rentas interiores mediante operaciones de intervención y sistemas de protección fronteriza. La PAC contribuyó al crecimiento económico y logró garantizar el suministro al consumidor europeo. Hasta mediados de la década de los noventa la PAC fue la política comunitaria más importante desde el punto de vista presupuestario. La Unión Europea (UE) se convirtió en el primer importador y el segundo exportador de productos agrícolas en el ámbito mundial. Sin embargo, el sistema, que respondía bien a una situación de déficit, generó problemas cuando se comenzaron a producir enormes excedentes de la mayoría de sus productos agrícolas. Surgieron tensiones en las relaciones con terceros países por los efectos sobre los precios de mercado internacionales de las exportaciones subvencionadas europeas (Heilbuth, 2004; Salazar y Franco, 2004). Las reformas que se introdujeron en la PAC en las décadas de los ochenta y noventa permitieron a la UE responder a las obligaciones que le exigían los acuerdos de la Ronda Uruguay del Acuerdo General sobre Aranceles Aduaneros y Comercio (GATT) y de la Organización Mundial del Comercio (OMC). Estos compromisos obligatorios suponían una reducción de 20\% del apoyo interior prestado a la agricultura, un recorte de $36 \%$ del gasto presupuestario destinado a la subvención de las exportaciones y otra disminución de $21 \%$ en el volumen de exportaciones subvencionadas.

La PAC ha sufrido tres grandes modificaciones: la Reforma de 1992, la Agenda 2000 y la Revisión Intermedia de 2003. En cada una de ellas se han introducido nuevos objetivos y diferentes medidas que permitieran encarar los retos que aparecieron y solventar las contradicciones existentes, lo que produjo una lenta transformación en la filosofía de la PAC originaria, que ha supuesto el nacimiento de dos nuevas políticas: la de desarrollo rural y la agroambiental. Las primeras directivas con carácter agroambiental fueron adoptadas en 1972, aunque entonces sólo incorporaron tímidamente algunos objetivos específicos.

Los pilares de la reforma de la PAC de junio de 2003 se basan en el desacoplamiento de las ayudas directas a los productores y en la introducción del régimen de pago único por explotación, lo que refuerza de esta manera el concepto de ecocondicionalidad gestado en la Agenda 2000. En consecuencia, la mayoría de las organizaciones comunes de mercado (OCM) adoptaron el nuevo sistema durante 2005 y 2006, excepto los nuevos Estados miembros, lo que redujo progresivamente las ayudas directas aún existentes hasta 2012 y condicionó su pago al cumplimiento de ciertas medidas agroambientales. Por otra parte, tanto las ayudas acopladas como desacopladas estarán sometidas a un mecanismo de modulación que consiste en la aplicación de un porcentaje de retención de tales ayudas (3\% en 2005, 4\% en 2006

Vol. 39, núm. 154, julio-septiembre / 2008

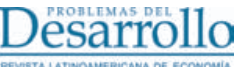


y $5 \%$ anual entre 2007 y 2012). Los importes retenidos se destinarán a financiar medidas agroambientales, de diversificación de la economía rural, etcétera. No obstante, algunos cultivos, entre los que se encuentra el olivar, recibirán una ayuda suplementaria para compensar la pérdida de ingresos provocada por la modulación y la transición al sistema de pago único. El pago único es una ayuda a la renta de los agricultores en forma de un pago anual que se calcula en función de los derechos adquiridos durante el periodo de referencia histórica 2000-2002, con el objeto de garantizar una mayor estabilidad de ingresos a los agricultores, quienes pueden decidir la cantidad que desean producir sin perder las ayudas, ajustando de este modo la oferta a la demanda del mercado. Para el caso del olivar se considera un periodo de referencia histórica ligeramente más amplio que el general, que abarca el cuatrienio 1999/2000-2002/2003.

El Reglamento 1782/2003 sobre condicionalidad de las ayudas directas se hace eco de la regulación de los mercados internacionales determinada por la Organización Mundial de Comercio tras la Ronda de Uruguay (1994), la cual establece la disminución de las ayudas directas a la producción como mecanismo de liberalización de los mercados agrarios. Esta normativa establece en el ámbito comunitario condiciones comunes para los pagos directos de las distintas medidas de apoyo a la renta previstas en la PAC, el cual será completado posteriormente por otras disposiciones normativas acerca de la aplicación de la condicionalidad, la modulación y el sistema integrado de gestión y control. El Reglamento 1782/2003, por tanto, subordina las ayudas directas en lo que se refiere a una serie de prácticas agroambientales relacionadas con el mantenimiento de las tierras en buenas condiciones agrarias y el respeto de las normas en materia de salud pública, sanidad animal y vegetal, ambiente y bienestar animal (ecocondicionalidad), realizándose dicho pago único con referencia a la explotación. También prevé la introducción de un sistema de modulación y disciplina financiera basado en la reducción progresiva de los pagos directos con objeto de lograr un mayor equilibrio entre los instrumentos destinados a promover una agricultura sostenible y los destinados a fomentar el desarrollo rural, de cara al periodo 2005-2012 (entre 3\% y 5\% anual, según el artículo 10), destinando así más fondos hacia el recién creado Fondo Europeo para la Agricultura y el Desarrollo Rural (FEADER), el cual cuenta con una dotación escasa para el periodo 2007-2013.

El artículo 3 del Reglamento 1782/2003 menciona los requisitos prioritarios agroambientales que debe cumplir un agricultor para recibir pagos directos por su explotación (cuadro 2).

\section{DeSTarrollo}


Cuadro 2

Buenas condiciones agrarias y ambientales

\begin{tabular}{ll}
\hline Erosión del suelo & - Cobertura mínima del suelo \\
& - Laboreo adaptado a la pendiente \\
& - Terrazas de retención \\
\hline Materia orgánica del suelo & - Normas en materia de rotación de cultivos \\
& - Gestión de rastrojos \\
\hline Estructura del suelo & - Utilización de maquinaria adecuada \\
\hline Nivel de mantenimiento & - Niveles mínimos de carga ganadera \\
& - Protección pastos permanentes \\
& - Mantenimiento particularidades topográficas \\
& - Prevención vegetación indeseable \\
\hline
\end{tabular}

Fuente: Anexo Iv, Reglamento 1782/2003.

Aunque las ayudas desacopladas se orientan hacia el productor y no hacia el producto, dirigiendo las producciones a la demanda de los mercados, estas ayudas presentan unos riesgos e incertidumbres relacionados, principalmente, con la forma de conectar multifuncionalidad y superficie, ayudas desvinculadas y hectáreas subvencionables con problemas específicos (por ejemplo, erosión), explotaciones con derecho a pagos directos y distorsión de la competencia, efectos sobre el empleo y desajustes en los mercados, complejidad de la gestión administrativa y consenso sobre los estándares ambientales mínimos, etcétera. Además, existen riesgos asociados al cumplimiento de las exigencias ecológicas debido al incremento de costos que supone su aplicación en las explotaciones más proclives a la erosión, que pueden motivar el abandono de tierras, pues los costos marginales de su aplicación son mayores en las explotaciones más erosionadas y cuya rentabilidad es menor (Varela-Ortega y Calatrava-Leyva, 2004).

\section{La economía de la erosión del suelo}

Los procesos erosivos han alcanzado la categoría económica de externalidades significativas en el proceso productivo (Cramb, 2001), pues históricamente los sistemas agrarios evolucionaron de forma paralela a dos factores básicos con incidencia directa sobre el mercado: por un lado, aumento de la presión demográfica, que implica mayores demandas de productos agrarios; y por otro, intensificación del uso de la tierra para aumentar la oferta agrícola. De tal forma que se ha pasado de sistemas agrarios forestales a sistemas de monocultivo anuales, con el consiguiente incremento en cada etapa de requerimientos en labores por hectárea y por trabajador. Con ello 
se logró generar e impulsar el avance de procesos erosivos de origen humano. La literatura económica sobre la erosión explica la existencia y persistencia de los procesos erosivos como consecuencia de procesos productivos intensivos no sostenibles en el terreno ambiental; además hay estudios sobre los factores que determinan la adopción de prácticas de conservación del suelo (PCS), y son muy numerosos a partir de la segunda mitad de la década de los noventa (cuadro 3). En estos trabajos de adopción dominan las investigaciones basadas en modelos de elección discreta, probit y logit (Pattanayak y Mercer, 1998; Valentín et al., 2004; Calatrava-Leyva et al., 2007).

Cuadro 3

Ejemplos de trabajos sobre adopción de prácticas de conservación del suelo

\begin{tabular}{|c|c|c|c|c|}
\hline Trabajos & Zona de estudio & Metodología & Cultivo & Efectos analizados \\
\hline Eaton (1996) & Malawi & Simulación & Maíz & Factores de la adopción \\
\hline Gretton y Salma (1997) & Australia & Modelo de regresión & Los de la zona & $\begin{array}{l}\text { Efectos de la erosión } \\
\text { sobre los beneficios }\end{array}$ \\
\hline $\begin{array}{l}\text { Pattanayak y Mercer } \\
\text { (1998) }\end{array}$ & Visayas(Filipinas) & Modelo probit & $\begin{array}{l}\text { Maíz, banana } \\
\text { y frutales }\end{array}$ & $\begin{array}{l}\text { Factores determinantes } \\
\text { de la adopción }\end{array}$ \\
\hline Dissart et al. (2000) & Québec (Canadá) & Programación lineal mixta & Maíz, cebada, soja & Costos de la adopción \\
\hline Wiig et al. (2001) & Tanzania & Modelo de equilibrio general & $\begin{array}{l}\text { Tabaco, café, } \\
\text { anacardo, maíz, etc. }\end{array}$ & $\begin{array}{l}\text { Efectos de política de ajuste } \\
\text { estructural sobre la erosión }\end{array}$ \\
\hline Vieth et al. (2001) & Sri Lanka & Análisis costo-beneficio & Té, tabaco & $\begin{array}{l}\text { Nivel de adopción con } \\
\text { iniciativa pública y privada }\end{array}$ \\
\hline Parra (2003) & Andalucía (España) & Multicriterio (AHP) & Olivar ecológico & Costos de la adopción \\
\hline Valentin et al. (2004) & Kansas (EU) & Modelos logit & $\begin{array}{l}\text { Maíz, trigo, } \\
\text { soja y sorgo }\end{array}$ & $\begin{array}{l}\text { Impactos de la adopción } \\
\text { sobre el beneficio }\end{array}$ \\
\hline Benítez et al. (2006) & Manabí(Ecuador) & Dominancia estocástica & Café, arroz, maíz. & $\begin{array}{l}\text { Efectos de la aversión } \\
\text { al riesgo }\end{array}$ \\
\hline Calatrava et al. (2007) & Andalucía(España) & Modelos probit & Olivar & Factores de la adopción \\
\hline
\end{tabular}

Fuente: Elaboración propia.

Tres cuestiones son relevantes en el estudio de esta problemática: la contaminación de las aguas por sedimentos, la falta de información sobre el valor económico del suelo, y la incapacidad de incorporar consideraciones a largo plazo en las decisiones relativas al uso del suelo (Wade y Heady, 1978; McConnell, 1983). En cuanto a la primera cuestión, es evidente que existen claros y cuantiosos costos para la sociedad que se derivan de la erosión. La conservación de suelos reduce las externalidades negativas y los daños off-site, tales como la reducción de la carga de sedimentos en 
ríos y lagos, daños químicos a la fauna piscícola, etcétera. Dichos beneficios para la sociedad pueden garantizar o justificar la conservación de los suelos, incluso cuando la rentabilidad privada de ésta no exista (Walker, 1982). La literatura sobre el tema ha analizado las condiciones bajo las cuales los óptimos privados y sociales coinciden o divergen (Azqueta, 1994).

La segunda cuestión es la falta de información sobre el valor económico del suelo. Así, se observa cómo en el mercado de la tierra se cotizan cada vez más los usos económicos no agrícolas (inmobiliarios, turísticos y especulativos, principalmente) con respecto a los agrícolas, primando en este mercado las preferencias asociadas a economías de escala de aquellos agentes económicos que mejoran a corto plazo el valor de la tierra agrícola, basadas en grandes superficies latifundistas y suelos con bajo riesgo de erosión y alta fertilidad (Cramb, 2001), al dar prioridad no sólo a la búsqueda de la eficiencia económica, sino al eliminar de su análisis económico toda consideración sobre la eficiencia ambiental. La hipótesis subyacente es que el mercado internaliza el problema erosivo en la medida que el valor de la tierra incorpore el nivel de conservación del suelo e incremente los costos privados del agricultor. Sin embargo, los problemas de medida de los costos de oportunidad de conservar el suelo no son fácilmente resolubles, especialmente porque son muy sensibles a la tasa de descuento utilizada (Van Kooten et al., 1989).

La tercera cuestión importante en el análisis económico de la erosión se refiere a la dimensión temporal de los efectos endógenos de la degradación de la tierra, ya que influye de forma decisiva en la toma de decisiones de los agricultores con respecto a la adopción de tecnologías de conservación del suelo. En particular, existe un desfase temporal a dos niveles: 1) Antes de la adopción, los efectos de la erosión son a largo plazo, igual que los beneficios derivados de la adopción de PCS, mientras que los costos de inversión en PCS son a corto plazo. 2) Después de la adopción, los costos de inversión en PCS (sin subvención) superan en el corto plazo los beneficios, que suelen distribuirse más dilatadamente en el tiempo, siendo, por lo general "beneficios ocultos o no percibidos" para el agricultor (Valentin et al., 2004). Este desfase temporal adquiere matices distintos según las características del agricultor y de su explotación.

Un elemento relacionado estrechamente con el horizonte temporal es la percepción de los agricultores sobre el problema de la erosión, sus costos y beneficios, clave para la adopción de prácticas de conservación de suelos. La evidencia empírica al respecto muestra que los agricultores son conscientes del problema, pero que en muchos casos no están preocupados por él. La principal razón es que existe la posi-

Vol. 39, núm. 154, julio-septiembre / 2008

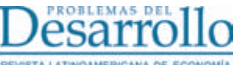


bilidad de sustituir el suelo por otros factores de producción (Wade y Heady, 1978). Ello hace que no se incorporen los beneficios a largo plazo en la función de utilidad de los agricultores, lo que lleva generalmente a una intensificación de los sistemas de producción (Lee, 1980).

Debido a la particular naturaleza económica del proceso de adopción de prácticas conservacionistas, suelen plantearse desde la teoría económica de la erosión diversos modelos de intervención pública que disminuyan, por un lado, los costos privados de los agricultores adoptantes y, por otro, el costo social neto en términos de menores pérdidas de suelo y menores costos económicos del programa de control de la erosión (Seitz et al., 1979; Feder, 1982; Deybe, 2004). Específicamente, las subvenciones públicas presentan un doble efecto, directo e indirecto, sobre la decisión de incumplimiento de los requisitos agroambientales exigidos; por un lado, el efecto directo implica que un incremento de las subvenciones alienta el incumplimiento puesto que aumentan los beneficios esperados; y, por otro, dicho aumento de las ayudas públicas desalienta el incumplimiento porque aumenta su costo de oportunidad, es decir, la oportunidad de obtener beneficios derivados de la adopción inmediata de prácticas conservacionistas (Giannakas y Kaplan, 2005).

\section{El caso del olivar en el sur de España}

La erosión es consustancial al olivar debido a varios factores intrínsecos, como pueden ser el cultivo en suelos en pendiente, el clima mediterráneo (que se caracteriza por la alternancia de épocas del año totalmente secas con otras de lluvias intensas), suelos arcillosos con baja velocidad de infiltración, escasa cobertura del suelo por la copa del olivo, y sistemas de cultivo que eliminan de manera sistemática la cubierta vegetal herbácea (Barranco et al., 2004). Todo lo cual supone que la erosión sea el principal problema ambiental en el olivar, y más específicamente en el olivar de secano.

Según las estadísticas del Ministerio de Agricultura, en 2007 la superficie olivarera española en plantación regular superó los dos millones y medio hectáreas (cercana a $10 \%$ de la superficie agrícola nacional y un cuarto de la superficie olivarera mundial), de las que $16 \%$ corresponde a explotaciones de regadío. Andalucía es la región española con mayor superficie dedicada al olivar, la cual representa casi $20 \%$ de la superficie geográfica andaluza. En los últimos años, la agricultura en esta región ha seguido un proceso de especialización productiva en el cultivo del olivar, que representa 37\% de la producción mundial en 2004-2005 y 43\% de la UE (García, 2005). Después de la crisis de la década de los setenta, el olivar andaluz se ha convertido 
en un sector rentable, que produce cerca de $70 \%$ del aceite de oliva español, ocupa la cuarta parte de las tierras labradas y se extiende, sobre todo, por las provincias de Jaén, Granada y Córdoba. De todo ello se deduce la importancia económica y ambiental de la lucha contra la erosión en este sector por medio de la promoción de buenas prácticas agrarias y la aplicación de medidas que alienten la adopción de prácticas de conservación del suelo. Recordemos que en España más de 50\% del suelo agrícola está clasificado con un riesgo medio-alto de erosión, y en Andalucía dicha cifra alcanza 70\% (González, 2003). La selección de las provincias de Granada y Jaén se debe al hecho de que son zonas donde predominan áreas montañosas de cultivo de olivar y, por ello, con un elevado riesgo de erosión, lo que permite una mejor delimitación de los efectos de degradación del suelo agrícola.

Existen tres tipos de producción en olivar: el sistema convencional, el ecológico y el integrado. En Andalucía y también en el resto de España se vienen desarrollando en los últimos años formas alternas de cultivo del olivar que intentan reducir las externalidades ambientales negativas, como la erosión, y aumentar al mismo tiempo la calidad alimentaria del producto. Se trata del olivar de cultivo ecológico y, más recientemente, el olivar de producción integrada (Calatrava-Requena, 2001). El olivar es el primer cultivo de agricultura ecológica en Andalucía, y también en el ámbito nacional (si excluimos pastos y praderas). El cultivo ecológico del olivar presenta dos limitaciones, su baja productividad y su elevado costo por unidad de producción. La baja productividad se debe a que el olivar ecológico se ha desarrollado históricamente en tierras que, en general, tienen bajos rendimientos para el olivar convencional. Por lo que respecta al costo de cultivo, el encarecimiento se debe al mayor costo de los abonos y los tratamientos fitosanitarios, ya que la mano de obra es similar. A pesar de ello, el olivar ecológico resulta sensiblemente más rentable que el convencional, puesto que el precio de la aceituna ecológica es superior al convencional. En relación con el olivar de producción integrada, en realidad se trata de un cultivo convencional "racionalizado" del olivar, en el que algunas prácticas ambientales más agresivas se han eliminado o reducido. Las perspectivas de obtener un diferencial positivo de precios en el mercado por el hecho de la producción integrada son inciertas (CalatravaRequena, 2001).

\section{Resultados del estudio empírico}

En este apartado se resumen y comentan los principales resultados obtenidos del análisis de los indicadores de eficiencia de las explotaciones de olivar encuestadas, así como del estudio de los factores que determinan la adopción individual de la técnica 
de no laboreo para la conservación de suelos y su proceso agregado de difusión temporal entre los agricultores de la zona. El tamaño muestral es de 223 explotaciones de olivar, con un total de 582 parcelas, cubriendo una superficie de 2,595 hectáreas. La realización de las encuestas y el trabajo de campo necesario para el análisis empírico de esta investigación fueron financiados con cargo al proyecto europeo sobre el futuro del olivar de montaña (Proyecto QLK5-CT-2002-01841). Se analiza la productividad conjunta, económica y ambiental de las provincias andaluzas de Granada y Jaén en 2004 (véase mapa 1), y se distinguen según las PCS, particularmente tres: laboreo según curvas de nivel, no laboreo con aplicación de herbicidas, y mantenimiento de bancales.

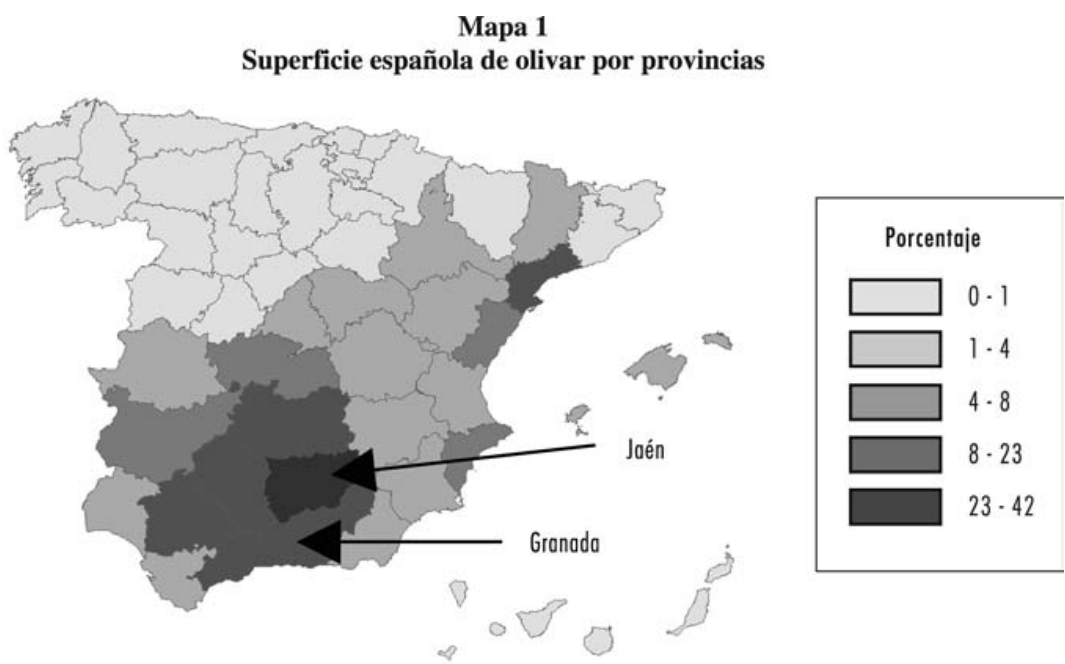

Fuente: Ministerio de Agricultura, Pesca y Alimentación, 2006.

Las explotaciones encuestadas fueron seleccionadas por sus niveles medios y altos de erosión del suelo. La información relativa a los costos de jornales de laboreo, poda, aplicación de herbicidas y fitosanitarios y recolección, fue estimada a partir de los datos estimados por la Consejería de Agricultura y Pesca de la Junta de Andalucía sobre el olivar andaluz (Junta de Andalucía, 2003). También se calcularon los ingresos por hectárea a partir de los precios oficiales de venta del kilogramo de aceituna (almazara), estableciendo un coeficiente de variación de 10\% para posibles variaciones respecto al precio medio ( 0.45 euros por kilogramo de aceituna en las últimas campañas).

\section{DeSarrollo}


Los principales indicadores calculados se refieren a las proporciones existentes entre costos e ingresos totales de cada una de las parcelas olivareras, por hectárea y árbol, según diferentes prácticas de conservación del suelo: laboreo siguiendo curvas de nivel, no laboreo con aplicación de herbicidas y mantenimiento de bancales. Asimismo, se estableció — previa consulta a expertos en el sector y en la zona- un intervalo para los costos que oscila entre $65 \%$ y $80 \%$ de los ingresos totales de la explotación. También se analizó si había diferencias según el régimen de cultivo, regadío o secano, y según la provincia, Granada o Jaén, y no se apreciaron resultados inesperados o incongruentes. Los cuadros siguientes resumen los principales estadísticos (media, desviación típica, mediana, moda, coeficientes de asimetría y de curtosis) para los cuatro indicadores considerados, siguiendo los supuestos antes mencionados de precio medio y estimaciones de los intervalos de costos, en relación con los ratios de productividad según las PCS (cuadros 4-10).

Cuadro 4

Estimación de ratios de productividad de parcelas de secano de Granada que realizan no laboreo con herbicidas

\begin{tabular}{lcccc}
\hline Estadísticos & Ingreso por ha & Ingreso por olivo & Intervalo costos totales por ha & Intervalo costos totales por olivo \\
\hline Media & 1153.85 & 10.68 & $750-923$ & $6.94-8.55$ \\
Desviación típica & 459.73 & 3.48 & $299-368$ & $2.26-2.79$ \\
Moda & 1350.00 & 9.00 & $878-1080$ & $5.85-7.20$ \\
Mediana & 1125.00 & 11.25 & $731-900$ & $7.31-9.00$ \\
Asimetría & 2.23 & -0.16 & 2.23 & -0.16 \\
Curtosis & 19.64 & 1.50 & 19.64 & 1.50 \\
\hline
\end{tabular}

Fuente: Elaboración propia a partir de la muestra.

Cuadro 5

Estimación de ratios de productividad de parcelas de secano de Jaén que realizan laboreo según curvas de nivel

\begin{tabular}{lcccc}
\hline Estadísticos & Ingreso por ha & Ingreso por olivo & Intervalo costos totales por ha & Intervalo costos totales por olivo \\
\hline Media & 1961.27 & 21.16 & $1275-1569$ & $13.75-16.93$ \\
Desviación típica & 1674.40 & 14.58 & $1088-1340$ & $9.48-11.66$ \\
Moda & 2250.00 & 22.50 & $1462-1800$ & $14.63-18.00$ \\
Mediana & 1597.50 & 19.02 & $1038-1278$ & $12.36-15.21$ \\
Asimetría & 3.87 & 1.50 & 3.87 & 1.50 \\
Curtosis & 23.34 & 4.27 & 23.34 & 4.27 \\
\hline
\end{tabular}

Fuente: Elaboración propia a partir de la muestra. 


\section{Cuadro 6}

Estimación de ratios de productividad de parcelas de regadío de Jaén que realizan no laboreo con herbicidas

\begin{tabular}{lcccc}
\hline Estadísticos & Ingreso por ha & Ingreso por olivo & Intervalo costos totales por ha & Intervalo costos totales por olivo \\
\hline Media & 2013.33 & 19.80 & $1309-1611$ & $12.87-15.84$ \\
Desviación típica & 221.12 & 2.49 & $144-177$ & $1.62-1.99$ \\
Moda & 2250.00 & 22.50 & $1463-1800$ & $14.63-18.00$ \\
Mediana & 1987.50 & 20.01 & $1292-1590$ & $13.00-16.01$ \\
Asimetría & -0.19 & -0.21 & -0.19 & -0.21 \\
Curtosis & -0.89 & -1.36 & -0.89 & -1.36 \\
\hline
\end{tabular}

Fuente: Elaboración propia a partir de la muestra.

\section{Cuadro 7}

Estimación de ratios de productividad de parcelas de regadío de Jaén que no realizan ninguna PCS.

\begin{tabular}{lcccc}
\hline Estadísticos & Ingreso por ha & Ingreso por olivo & Intervalo costos totales por ha & Intervalo costos totales por olivo \\
\hline Media & 2105.48 & 14.77 & $1369-1684$ & $9.60-11.81$ \\
Desviación típica & 1401.02 & 7.62 & $911-1121$ & $4.95-6.09$ \\
Moda & 2250.00 & 4.50 & $1463-1800$ & $13.16-16.20$ \\
Mediana & 2020.50 & 18.00 & $1313-1616$ & $11.70-14.40$ \\
Asimetría & 3.23 & -0.57 & 3.23 & -0.57 \\
Curtosis & 11.91 & -1.63 & 11.91 & -1.63 \\
\hline
\end{tabular}

Fuente: Elaboración propia a partir de la muestra.

Cuadro 8

Estimación de ratios de productividad de parcelas de regadío de Granada que realizan laboreo según curvas de nivel

\begin{tabular}{lcccc}
\hline Estadísticos & Ingreso por ha & Ingreso por olivo & Intervalo costos totales por ha & Intervalo costos totales por olivo \\
\hline Media & 2093.45 & 16.49 & $1361-1675$ & $10.72-13.19$ \\
Desviación típica & 1794.10 & 15.39 & $1166-1435$ & $10.01-12.32$ \\
Moda & 2250.00 & 22.50 & $1463-1800$ & $14.63-18.00$ \\
Mediana & 1650.00 & 13.88 & $1072-1320$ & $9.02-11.10$ \\
Asimetría & 1.47 & 3.76 & 1.47 & 3.76 \\
Curtosis & 1.74 & 20.88 & 1.74 & 20.88 \\
\hline
\end{tabular}

Fuente: Elaboración propia a partir de la muestra.

\section{Desaarrollo}


Cuadro 9

Estimación de ratios de productividad de parcelas de regadío de Granada que no realizan ninguna PCS.

\begin{tabular}{lcccc}
\hline Estadísticos & Ingreso por ha & Ingreso por olivo & Intervalo costos totales por ha & Intervalo costos totales por olivo \\
\hline Media & 2239.02 & 13.32 & $1455-1791$ & $8.66-10.66$ \\
Desviación típica & 2429.76 & 8.21 & $1579-1943$ & $5.33-6.56$ \\
Moda & 1800.00 & 9.00 & $1170-1440$ & $7.37-11.88$ \\
Mediana & 1800.00 & 11.25 & $1170-1440$ & $7.31-9.00$ \\
Asimetría & 3.97 & 1.57 & 3.97 & 1.57 \\
Curtosis & 18.43 & 4.42 & 18.43 & 4.42 \\
\hline
\end{tabular}

Fuente: Elaboración propia a partir de la muestra.

Cuadro 10

Estimación de ratios de productividad de todas las parcelas que realizan mantenimiento de bancales

\begin{tabular}{lcccc}
\hline Estadísticos & Ingreso por ha & Ingreso por olivo & Intervalo costos totales por ha & Intervalo costos totales por olivo \\
\hline Media & 1711.44 & 12.31 & $1112-1369$ & $8.00-9.85$ \\
Desviación típica & 1255.97 & 7.33 & $816-1005$ & $4.76-5.86$ \\
Moda & 1080.00 & 11.25 & $702-864$ & $7.31-9.00$ \\
Mediana & 1175.63 & 10.55 & $764-941$ & $6.86-8.44$ \\
Asimetría & 1.72 & 2.14 & 1.72 & 2.14 \\
Curtosis & 1.89 & 4.74 & 1.89 & 4.74 \\
\hline
\end{tabular}

Fuente: Elaboración propia a partir de la muestra.

En síntesis, los principales resultados son los siguientes: una especialización de las parcelas granadinas de secano en la técnica de no laboreo con herbicidas (cuadro 4). Una especialización de las parcelas de secano de Jaén en la técnica de laboreo según curvas de nivel (cuadro 5). Una especialización de las parcelas de regadío de Jaén en la técnica de no laboreo con herbicidas (cuadro 6). Una especialización de las parcelas de regadío de Granada en la técnica de laboreo según curvas de nivel (cuadro 8).

De los cuadros 4 y 5 se desprende que las parcelas de secano de Jaén que aplican laboreo según curvas de nivel son más rentables (en más de 222 euros de margen por hectárea) que las de secano de Granada que realizan no laboreo con aplicación de 
herbicidas. Si se comparan técnicas de conservación del suelo, por ejemplo, en las parcelas de regadío de Jaén, entre las que aplican no laboreo con herbicidas y las que no aplican ninguna PCS (cuadros 6 y 7), se observa que en promedio hay un ligero margen neto superior en las parcelas que no realizan ninguna técnica de conservación (26 euros por hectárea). Si bien ocurre al contrario cuando se considera la media del margen neto por olivo, se supone cercano a 1.30 euros más en las parcelas de regadío que aplican no laboreo con herbicidas, con respecto a las que no realizan ninguna técnica de conservación. De forma similar, puede realizarse la misma comparativa con respecto a las explotaciones de regadío de Granada que aplican laboreo según curvas de nivel y las que no realizan ninguna PCS (cuadros 8 y 9). El margen neto medio por hectárea es superior en casi 40 euros más en las explotaciones que no realizan ninguna PCS. Mientras que el margen neto medio por árbol es superior en las explotaciones granadinas de regadío que realizan laboreo según curvas de nivel en aproximadamente 90 céntimos de euro más que en las que no realizan ninguna PCS. El cuadro 10 resume las principales medidas estadísticas de los ratios asociados al total de parcelas en las dos provincias que declaran realizar mantenimiento de bancales. Se observa cómo estas parcelas presentan una distribución ligeramente asimétrica a la derecha y un moderado apuntamiento. Además el margen neto medio por hectárea es de más de 470 euros, mientras que el promedio del margen neto por olivo oscila entre 2.46 y 4.31 euros.

De especial importancia para el control de la erosión agraria es el sistema de laboreo o labranza utilizado. El laboreo cumple tres funciones principales. En primer lugar, prepara el suelo para la adecuada emergencia y vigoroso desarrollo del cultivo. En segundo, controla las malas hierbas que compiten con el cultivo por el agua, la luz y los nutrientes. Finalmente, mejora la estructura del suelo, lo que favorece la infiltración del agua, reduciendo la escorrentía y por tanto la erosión. No existe ningún tipo de laboreo o labranza que evite totalmente la erosión (Barranco et al., 2004). Sin embargo, estas prácticas alcanzan una mayor efectividad cuando se combinan con otras como el laboreo siguiendo las curvas de nivel o la construcción de bancales, preferentemente en zonas de montaña (Calatrava-Leyva et al., 2007).

El porcentaje de agricultores, cuya actitud ante el riesgo de adoptar una innovación técnica es "prudente" o "muy prudente", supera las tres cuartas partes de los encuestados, lo cual tiene concordancia con su percepción sobre la erosión, distinguiéndose claramente la "percepción general del problema" de la "percepción del problema en la propia explotación". Así, mientras que 92\% considera que en términos generales la erosión es un problema grave o muy grave, dicho porcentaje se reduce en un tercio cuando se toman en cuenta los efectos erosivos en la propia finca, 
lo cual contrasta con la realidad del problema en la zona. Para profundizar en este aspecto se ha estimado un modelo probit de adopción de la principal PCS adoptada entre los olivicultores, el no laboreo con aplicación de herbicidas. La selección de las variables relacionadas con esta práctica se realizó mediante un test Chi-cuadrado, donde se excluyen del análisis las variables no relacionadas. En el cuadro 11 se observan los coeficientes de las variables explicativas, así como los efectos marginales y elasticidades. El nivel de ajuste del modelo es relativamente alto ( $75.48 \%$ de predicciones correctas y un seudo- $\mathrm{R}^{2}$ bastante bueno para este tipo de modelos, $20.62 \%$ ). La probabilidad de que el agricultor adopte la práctica de no laboreo con herbicidas aumenta de manera significativa con dos factores: el adoptante sólo emplea mano de obra familiar en la explotación y lleva contabilidad sólo con fines impositivos y no con propósitos de gestión. Mientras que la probabilidad de adoptar esta técnica disminuye cuando el agricultor tiene más de 60 años.

Cuadro 11

Modelo probit de adopción de no laboreo con herbicidas

\begin{tabular}{|c|c|c|c|}
\hline Variables binarias explicativas & Coeficientes & Efectos marginales & Elasticidades \\
\hline CONSTANIE & $-0.9831\left(^{* \star)}\right.$ & $-0.3146(* \star *)$ & \\
\hline INNOV (alto nivel de adopción) & -0.3284 (n.s.) & -0.1449 (n.s.) & -0.0439 \\
\hline MO (sólo mano de obra familiar) & $0.9774\left(^{(\star \star \star}\right)$ & $\left.0.21911^{\star \star \star}\right)$ & 0.2914 \\
\hline MAQ (uso de maquinaria propia) & -0.1967 (n.s.) & -0.0433 (n.s.) & -0.0401 \\
\hline PRET (contabilidad con fines impositivos) & $0.9444(* \star *)$ & $0.3399\left(^{\star \star \star}\right)$ & 0.3853 \\
\hline El (edad del agricultor inferior a 40 años) & 0.2313 (n.s.) & 0.0987 (n.s.) & 0.0687 \\
\hline \multicolumn{4}{|l|}{ E2 (edad entre 41 y 50 años) } \\
\hline E3 (edad entre 51 y 60 años) & 0.0118 (n.s.) & 0.0415 (n.s.) & 0.0193 \\
\hline E4 (edad superior a 60 años) & $-0.8123(\star \star)$ & $\left.-0.2212^{(\star \star}\right)$ & -0.0651 \\
\hline Núm. de observaciones & & 155 & \\
\hline Razón de verosimilitud & & 49.97 & \\
\hline Nivel de significación & & $0.00\left(^{\star \star \star}\right)$ & \\
\hline Seudo-Rs de McFadden & & $20.62 \%$ & \\
\hline Predicciones correctas & & $75.48 \%$ & \\
\hline
\end{tabular}

Nivel de significación: ${ }^{* *} p \leq 0.05,{ }^{* \star *} p \leq 0.01$

Nota: Estimación realizada con el paquete econométrico LIMDEP v.8.0.

Finalmente, se analizó el proceso de difusión seguido en el tiempo por los agricultores adoptantes de esta práctica (Mansfield, 1961; Fourt y Woodlock, 1960). En la gráfica 1 se puede ver el buen ajuste realizado mediante la estimación de la función 
Gráfica 1

Estimación del proceso de difusión de la técnica de no laboreo con aplicación de herbicidas en la zona de estudio

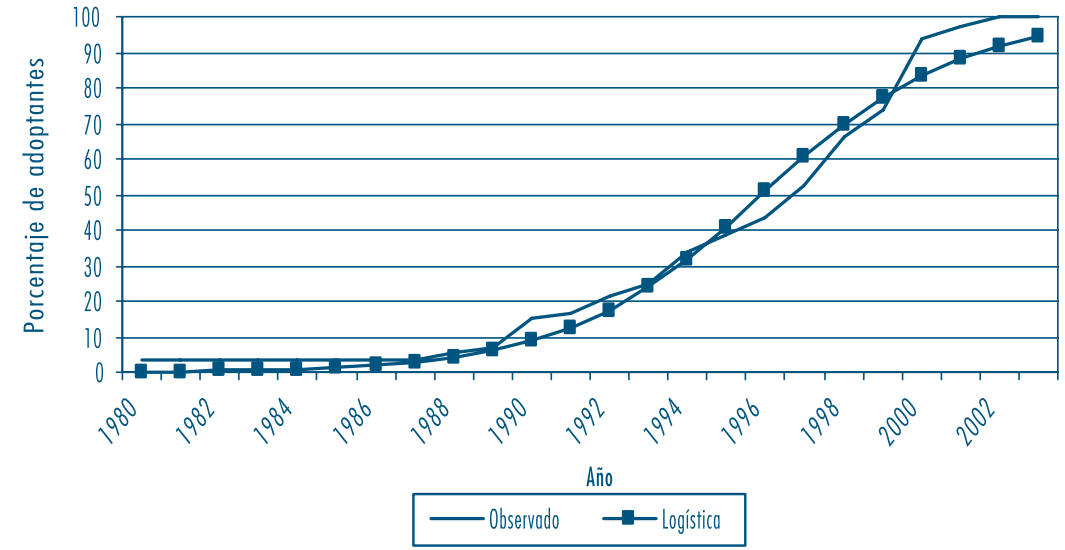

Fuente: Elaboración propia a partir de los datos de la muestra. Estimación realizada con SPSS v.14.

Gráfica 2

Estimación de pérdidas de suelo en Andalucía (1976-2003)

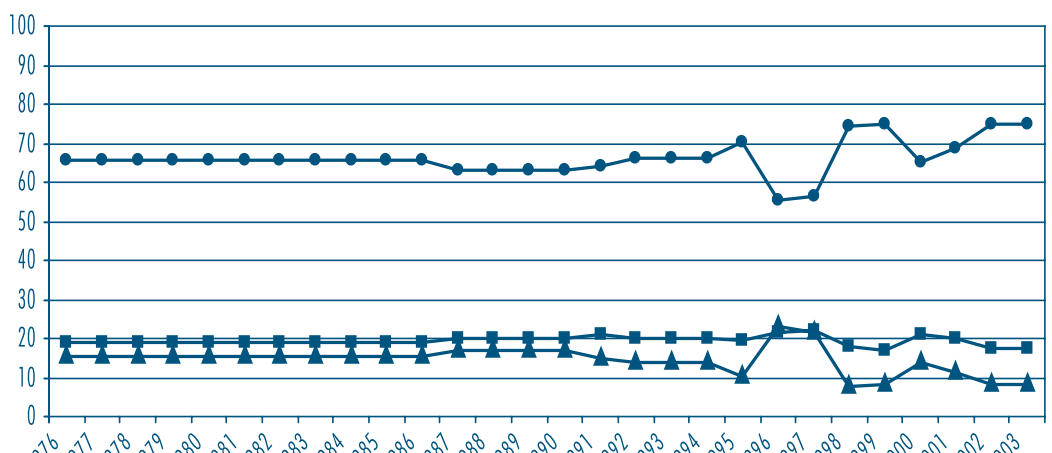

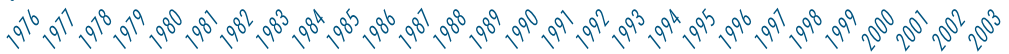

$$
\rightarrow \text { Bajas } \rightarrow \text {-Moderados } \rightarrow \text {-Altos }
$$

Fuente: Junta de Andalucía (2003).

logística (con el método de programación cuadrática secuencial y un techo de restricción no superior a $100 \%): N(t)=M /(1+\exp (a-b * t))=100 /(1+\exp (6,72-0,40 * \mathrm{t}))$. Donde $N(t)$ es el porcentaje acumulado de adoptantes en el tiempo $t, M$ el techo o nivel de saturación, $a$ la constante de integración y $b$ la tasa de difusión del proceso o co-

\section{Desaarrollo}


eficiente de imitación. El R² es de $98 \%$ y el nivel de saturación en términos absolutos corresponde a 113 agricultores, por lo que el nivel de penetración en el mercado de esta técnica sería el siguiente: por cada $1 \%$ de agricultores adoptantes son "contagiados" cada año $0.40 \%$ de los agricultores que aún no han adoptado; ello significa que por cada agricultor adoptante son persuadidos cada año $0.35 \%$ de agricultores que aún son no adoptantes. Se trata de un proceso en el que el "efecto imitación o contagio" es la principal fuente de incrementos en el porcentaje total acumulado de adoptantes (en contraposición a los modelos exponenciales que explican mejor el comportamiento de los procesos basados en influencias externas al sistema social en el que se desarrolla la adopción). Esta práctica tuvo su despegue en los años ochenta y alcanzó su etapa de expansión en los noventa, estabilizándose a finales de esta década. Si comparamos este resultado con las estimaciones oficiales de la Junta de Andalucía (2003) sobre la clasificación de las tierras agrarias según niveles de pérdidas de suelo, resulta llamativa la coincidencia de la etapa de expansión de la técnica de no laboreo con los años en los que aumenta el porcentaje de tierras afectadas por niveles altos de pérdida de suelo (gráfica 2).

\section{Conclusiones}

A continuación se destacan las conclusiones básicas relativas a la revisión de la legislación europea sobre conservación del suelo, así como de la literatura económica sobre los efectos de la erosión en los suelos agrarios. Este análisis es fundamental para una gestión eficiente económica y ambiental de los suelos agrarios mediterráneos, pues éstos no son ajenos a los efectos del desarrollo económico; más aún, son uno de los principales recursos agrarios y ambientales más propensos a la degradación debido a usos intensivos y preponderantemente productivistas. Así, se ha visto que la legislación europea pretende avanzar desde una lógica subvencionista "incondicionada" hacia una lógica subvencionista de condicionamiento de ayudas económicas al cumplimiento de una serie de requisitos agroambientales, previéndose en el futuro una normativa más restrictiva que penalice los comportamientos económicos no sostenibles desde la perspectiva ambiental. No obstante, y en consecuencia, en la medida que las subvenciones ambientales constituyan un mecanismo para canalizar subsidios al sector se estará alentando un comportamiento económico ineficiente (desde un punto de vista social y en un contexto mundial de globalización económica), y en términos ambientales ambiguo como se deduce del estudio empírico realizado.

Por otra parte, en lo referente a los antecedentes de investigaciones previas sobre análisis económicos de los efectos de la erosión del suelo sobre la productividad de la 
tierra y su conservación a largo plazo, se observa cómo tres cuestiones son transversales en el fenómeno erosivo: la contaminación de los recursos hídricos por acumulación de sedimentos, la necesidad de información para valorar económicamente los recursos edáficos, y la influencia del tiempo sobre las decisiones relativas a la gestión racional del suelo. En particular, en la región mediterránea se han realizado varios trabajos sobre la adopción de PCS, de los que destacan los realizados recientemente en España sobre el cultivo del olivar, si bien son pocos los que se han centrado en un análisis de la productividad global derivada de la aplicación de técnicas conservacionistas.

Las principales conclusiones del análisis empírico realizado en este trabajo sobre explotaciones de olivar de las provincias de Granada y Jaén en 2004 son las que a continuación se detallan: el margen neto medio por hectárea en las parcelas de regadío en las dos provincias es mayor que en las parcelas de secano. En la provincia de Jaén las parcelas de regadío son en promedio más rentables que las de secano, mientras que en la provincia de Granada las diferencias de rentabilidad entre parcelas de regadío y secano son menos acusadas. Se observa una proporción mayor de parcelas granadinas de secano que emplean la técnica de no laboreo con herbicidas, frente a las de Jaén que utilizan la técnica de laboreo según curvas de nivel. Y en contraposición a las parcelas de regadío de Granada que mayoritariamente realizan laboreo según curvas de nivel, frente a las jiennenses que hacen no laboreo con herbicidas. No obstante, los márgenes de productividad en las explotaciones encuestadas están más asociados al régimen de cultivo que a la adopción de PCS concretas; así, las explotaciones con sistemas de producción más intensivos, las de regadío, son más rentables y parecen más adaptadas y proclives a la adopción de ciertas PCS que reducen los efectos de la erosión, sobre todo a partir de los beneficios esperados (complementarios y adicionales a los beneficios propios de la producción) del cumplimiento de los requisitos agroambientales de la reciente legislación europea. Este resultado se ve confirmado por las estimaciones oficiales sobre pérdidas de suelo en Andalucía, observándose cómo en la etapa de expansión de la principal técnica de conservación de suelos en la zona (no laboreo con aplicación de herbicidas) aumentó paradójicamente el porcentaje de tierras afectadas por niveles altos de pérdida de suelo. 


\section{Bibliografía}

Arteaga, O., G. Hoffman, H. Schubart y H.D Schultz, "Investigaciones geológicas y arqueológicas sobre los cambios de la línea costera en el litoral de la Andalucía mediterránea", en Anuario Arqueológico de Andalucía, vol. 1, 1987, pp. 117-122.

Azqueta, D., Valoración económica de la calidad ambiental, Madrid, McGraw-Hill, 1994.

Barranco, D., R. Fernández-Escobar, R. y L. Rallo, El cultivo del olivo, Madrid, MundiPrensa y Junta de Andalucía, 2004.

Benítez, P. C., T. Kuosmanen, R. Olschewski, y G. C. Van Kooten, "Conservation payments under risk: a stochastic dominante approach", en American Journal of Agricultural Economics, vol. 88, núm. 1, 2006, pp. 1-15.

Calatrava-Leyva, J., J. A. Franco y M. C. González, "Analysis of the adoption of soil conservation practices in olive groves: the case of mountainous areas in southern Spain", en Spanish Journal of Agricultural Research, vol. 5, núm. 3, 2007, pp. 249-258.

Calatrava-Requena, J., M. C. González y N. Cabello, "Factores de innovación en olivar", Cáceres, Congreso Ibérico de Ciencias Hortícolas, mayo, 2001.

Consejo de la UE, Reglamento 1782/2003 de 29 de septiembre, Diario Oficial de la UE, Bruselas, Unión Europea, 2003.

Cramb, R. A., Soil Conservation Technologies for smallholder farming systems in the Philippine uplands: a socioeconomic evaluation, Canberra, ACIAR, 2001.

Deybe, D., "Cross-compliance assessment: modelling and policy analysis", Seminar of Evaluation of Cross-compliance, Granada, 19-20 de abril de 2004.

Dissart, J., L. Baker y P. Thomassin, “The economics of erosion and sustainable practices: the Saint-Esprit watershed", en Canadian Journal of Agricultural Economics, núm. 48, 2000, pp. 103-122.

Eaton, D., "The economic of soil erosion: a model of farm decision-making", Discussion Paper 96-01, International Institute for Environment and Development, Londres, 1996.

Feder, G., "Adoption of interrelated agricultural innovations: Complementarity and the impacts of risk, scale, credit", en American Journal of Agricultural Economics, núm. 64, 1982, pp. 94-101.
Fourt, L. y J. Woodlock, "Early prediction of market success for new grocery products", en Journal of Marketing, núm. 25, 1960, pp. 31-38.

García, D., "La rentabilidad económica de la industria agroalimentaria en el mercado del aceite de oliva. El caso de Andalucía”, en Agroalimentaria, núm. 21, 2005, pp. 43-55.

Giannakas, K. y J. D. Kaplan, "Policy design and conservation compliance on highly erodible lands", en Land Economics, vol. 81, núm. 1, 2005, pp. 20-33.

Gilman, A. y J. B. Thornes, Land-use and Prehistory in South-East of Spain, Londres, George Allen \& Unwin, 1985.

González, E., Erosión. La importancia de la conservación del suelo, Córdoba, Asociación Agricultura de Conservación, 2003.

Gretton, P. y U. Salma, "Land degradation links to agricultural output and profitability", en Australian Journal Agricultural and Resource Economics, núm. 4, 1997, pp. 209-224.

Heilbuth, P. E., Nailed to the bottom (La poderosa agricultura europea), Documental de TV, Canal de Historia, Digital +, 2004, http://video.google.es/videoplay?docid=7 $778052604868438850 \& \mathrm{q}=$ documental $+\mathrm{d}$ uration $\% 3$ Along \& total $=889 \&$ start $=10 \& \mathrm{n}$ $\mathrm{um}=10 \& \mathrm{so}=0 \&$ type $=$ search $\&$ plinde $=8$

Junta de Andalucía, El olivar andaluz, Sevilla, Unidad de Prospectiva de la Consejería de Agricultura y Pesca, 2003.

Lee, L. K., "The Impact of Landownership Factors on Soil Conservation", en American Journal of Agricultural Economics, vol. 62, núm. 5, 1980, pp. 1070-1076.

Mansfield, E., "Technical change and the rate of imitation”, en Econometrica, núm. 29, 1961, pp. 741-766.

MAPA, "Mapa de superficie de olivar por provincias", Madrid, Ministerio de Agricultura, Pesca y Alimentación, 2006.

McConnell, K. E., "An Economic Model of Soil Conservation", en American Journal Agricultural Economics, vol. 65, 1983, pp. 83-89.

Parra, C., "Sistemas de producción ecológica, integrada y convencional en olivar: estudio de difusión de innovaciones y evaluación multifuncional", tesis doctoral, Córdoba, 2003. 
Pattanayak, S. y D. E. Mercer, "Valuing soil conservation benefits of agroforestry, contour hedgerows in the Eastern Visayas, Philippines", en Agricultural Economics, núm. 18, 1998, pp. 31-46.

Pimentel, D., C. Harvey, P. Resosudarmo, K. Sinclair, D. Kurz, M. Mcnair, S. Crist, L. Shpritz, L. Fitton, R. Saffouri y R. Blair, "Environmental and economic cost of soil erosion and conservation benefits", en Science, núm. 267, 1995, pp. 1117-1123.

Salazar, M. y J. A. Franco, "Grupo de Cairns: la liberalización del comercio agrario (1986-2004)", Valencia, Foro Mundial sobre la Reforma Agraria, 4-8 diciembre, 2004.

Seitz, W. D., C. R. Taylor, R. Spitze, C. Osteen y M. C. Nelson, "Economic impacts of soil erosion control", en Land Economics, núm. 55, 1979, pp. 28-42.

Valentin, L., D. J. Bernardo y T. L. Kastens, "Testing the empirical relationship between Best Management Practice Adoption and Farm Profitability", en Review of Agri- cultural Economics, vol. 26, núm. 4, 2004, pp. 489-504.

Van Kooten, G. C., W. P. Weisensel y E. Jong, "Estimating the costs of soil erosion in Saskatchewan", en Canadian Journal of Agricultural Economics, núm. 37, 1989. pp. 63-75.

Varela-Ortega, C. y J. Calatrava-Leyva, "Evaluation of cross compliance: perspectives and implementation", Report of Seminar 4, Granada, 19-20 de abril de 2004.

Vieth, G. R., H. Gunatilake y L. J. Cox, "Economics of soil conservation: the upper Mahaweli watershed of Sri Lanka", en Journal of Agricultural Economics, vol. 52, 2001, pp. 139-152.

Wade, J. C. y E. O. Heady, "Measurement of Sediment Control Impacts on Agriculture", en Water Resources Research, vol. 14, núm. 1, 1978, pp. 1-8.

Walker, D. J., "A Damage Function to Evaluate Erosion Control Economics", en American Journal of Agricultural Economics, vol. 64 , núm. 4, 1982, pp. 690-698. 